\title{
Genes of NBS-LRR superfamily in Solanum phureja Juz. et Buk.
}

\author{
Gurina A.A. \\ Federal Research Centre "N.I. Vavilov All-Russian Institute of Plant Genetic Resources", \\ St. Petersburg, Russia \\ email: a.gurina@vir.nw.ru
}

Nucleotide-binding site (NBS) and leucine-rich repeat (LRR) genes are a huge superfamily of genes coding for the majority of resistance genes. The most predominant disease resistance genes cloned to date are the NBS-LRR resistance genes. We decided to analyze the persistence of different NBS-LRR genes in a group of 9 samples of Solanum phureja Juz. et Buk. For research we use SRA data from NCBI (PRJNA394943 project). We use sequences of 400 known potato NBS-LRR genes and 30 genes of another species of Solanaceae for alignment. Alignment was made by Burrows-Wheeler Aligner. Further we count coverage of each position in each gene using bedtools v2.29.0. As NBS-LRR genes contain conservative domains, only high coverage of the whole gene could be evidence of gene persistence. Previously known that resistance genes in general are not found in all samples of species, and It was shown for several genes. We characterized 9 sample of Solanum phureja across the entire set of known NBS-LRR genes. 116 genes are common for all samples, include Rpi-bt1 and $R G A-2(R B)$ resistance genes from Solanum bulbocastanum Dun. Presents of other 254 genes varied between samples. Also we find homologues of $\mathrm{Mi}-1$ NBS-LRR genes of Solanum lycopersicum L. which confers isolate-specific resistance against root-knot nematodes. 60 genes are not detected among studied samples. Interesting that two of the samples (SRX4645207 and SRX4645199) strongly differ from other samples and from each other. It could be related with geographic distribution of samples, because they were collected far from other samples, but to confirm this assumption we should test more samples, which would be one of our further objectives. Also we are going to expand the set of genes on all known resistance genes of Solanaceae, it allows to find homologues of resistance genes known for other crops (for example tomato or tobacco) and use them for potato selection. Acknowledgements: This work was supported by grants from the Russian Foundation for Basic Research (Project 20-516-10001 KO_a). 